\title{
Coulomb finite-size effects in quasi-2D systems
}

\author{
B Wood $\dagger$, W M C Foulkes $\dagger$, M D Towler $\ddagger$ and N D Drummond $\ddagger$ \\ $\dagger$ Blackett Laboratory, Imperial College, Prince Consort Road, London SW7 2BW, \\ United Kingdom \\ ¥ Theory of Condensed Matter Group, Cavendish Laboratory, Madingley Road, \\ Cambridge CB3 0HE, United Kingdon \\ E-mail: m.foulkes@imperial.ac.uk
}

\begin{abstract}
The Model Periodic Coulomb interaction [1] is a replacement for the Ewald sum, developed to reduce finite-size errors in the simulation of extended 3D systems. We investigate the generalization of this technique to quasi-2D systems; we show through testing in quantum Monte Carlo simulations that while the new interaction reduces the calculation time dramatically it does not reduce finite-size errors. We explain this by analyzing the finite-size errors generated when using the Ewald sum.
\end{abstract}

Submitted to: J. Phys.: Condens. Matter

PACS numbers: $02.70 . S s, 71.15 . N c, 73.20 .-\mathrm{r}$

\section{Introduction}

The current practical limit on the number of electrons in a quantum Monte Carlo (QMC) simulation is of the order of thousands. In order to simulate extended systems, it is therefore necessary to use a supercell with periodic boundary conditions. This device introduces finite-size errors. One source of these errors is the way in which the Coulomb energy is calculated. Traditionally, the Coulomb potential due to one electron (and the corresponding lattice of periodically-repeated images) was evaluated using the Ewald sum [2], which gives the periodic solution to Poisson's equation. However, it has been shown (in the case of 3D periodicity) that using the Ewald sum leads to an error in the energy per electron of order $1 / N$, where $N$ is the number of electrons [3]. The Modified Periodic Coulomb interaction [1] was introduced to avoid this. In addition to reducing the finite-size error, it also brings a considerable speed improvement.

In terms of calculating the Coulomb energy, quasi-2D systems provide a greater challenge than those with full 3D periodicity. Although the generalization of the Ewald potential to quasi-2D systems is known [4,5] (a more convincing derivation of this than the authors were able to find in the literature is given in Appendix A), it is awkward 

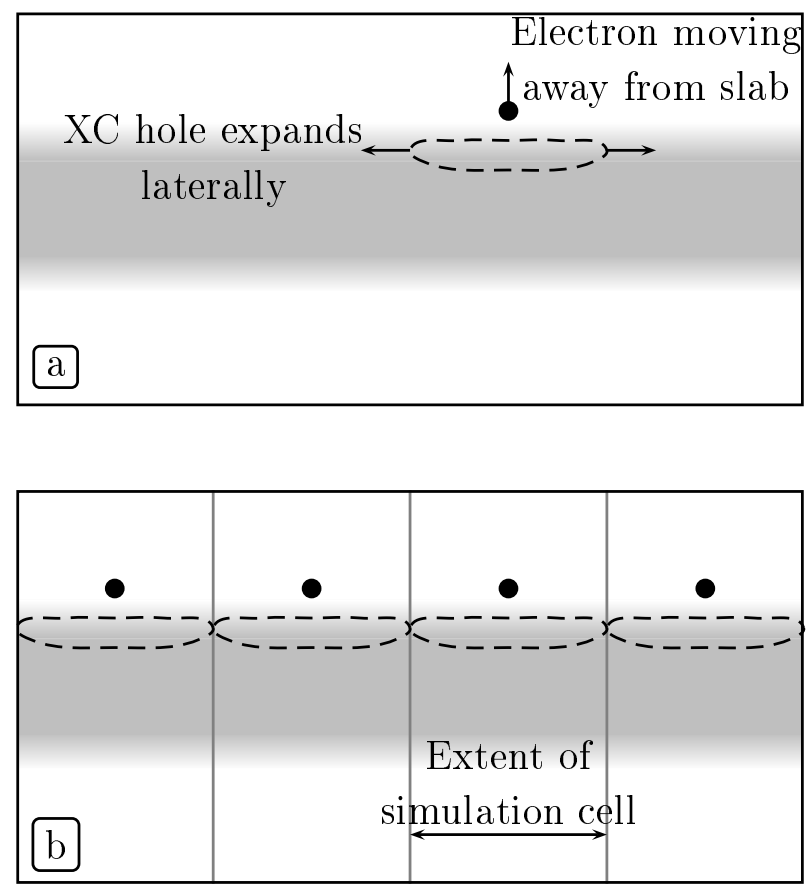

Figure 1. (a) A slab system with one electron far outside the slab, showing the associated exchange-correlation hole; (b) The result of using the Ewald interaction in this case. The shading represents the electron density.

and slow to evaluate. As in 3D, the quasi-2D Ewald sum gives the potential due to a lattice of charges, and as in 3D this produces finite-size effects.

To visualize a situation in which the quasi-2D Ewald potential is undesirable, consider a slab system where during the course of a simulation a single electron has moved some distance outside the slab (Figure 1(a)). The exchange-correlation hole associated with this electron remains largely within the slab, expanding laterally as the electron moves further away. When the Ewald sum is used for the electron-electron interaction, this configuration of electron and hole is repeated identically in every cell (Figure 1(b)); the result is that the electron interacts with an unphysical capacitor-like array of charges, and the interaction energy increases linearly with the distance outside the slab.

Another good reason exists for wanting to improve the accuracy of quasi-2D supercell simulations. Recent studies [6] (using non-QMC methods) of the simplest of quasi-2D systems, a slab of electron gas, have given results for the surface energy in disagreement with those obtained using QMC [7, 8]. It is important to test the possible sources of error in previous QMC calculations in order to demonstrate the suitability of QMC as a tool for studying surfaces.

In this paper, we implement and test the quasi-2D MPC interaction. We find that although finite-size errors are not reduced, the time required to evaluate the MPC interaction is around two orders of magnitude less than that for the Ewald sum. 


\section{The Model Interaction}

Under the Ewald scheme, the electron-electron part of the Coulomb energy is

$$
E_{\mathrm{e}-\mathrm{e}}^{\mathrm{EW}}=\left\langle\sum_{i>j} v_{\mathrm{E}}\left(\boldsymbol{r}_{i j}\right)\right\rangle+\frac{1}{2} N \xi
$$

where $N$ is the number of electrons in the simulation supercell and $v_{\mathrm{E}}\left(\boldsymbol{r}_{i j}\right)$ is the potential at $\boldsymbol{r}_{i}$ due to the charge at $\boldsymbol{r}_{j}$ and the corresponding periodically-repeated images, evaluated using the Ewald method. The self-interaction $\xi$ is the energy of interaction between a charge and its own images. Expressions for $v_{\mathrm{E}}$ and $\xi$ are derived in Appendix A. Here and throughout, Hartree atomic units are used.

The one- and two-particle and exchange-correlation-hole densities are defined in the usual way:

$$
\begin{aligned}
n(\boldsymbol{r}) & =\int_{\Omega} \mathrm{d} \boldsymbol{X}|\Psi(\boldsymbol{X})|^{2} \sum_{i} \delta\left(\boldsymbol{r}-\boldsymbol{r}_{i}\right) \\
n\left(\boldsymbol{r}, \boldsymbol{r}^{\prime}\right) & =\int_{\Omega} \mathrm{d} \boldsymbol{X}|\Psi(\boldsymbol{X})|^{2} \sum_{i \neq j} \delta\left(\boldsymbol{r}-\boldsymbol{r}_{i}\right) \delta\left(\boldsymbol{r}^{\prime}-\boldsymbol{r}_{j}\right) \\
& =n(\boldsymbol{r}) n\left(\boldsymbol{r}^{\prime}\right)+n(\boldsymbol{r}) n_{\mathrm{XC}}\left(\boldsymbol{r}, \boldsymbol{r}^{\prime}\right)
\end{aligned}
$$

where $\boldsymbol{X}$ represents all spatial and spin coordinates $\left(\boldsymbol{r}_{1} \sigma_{1} \ldots \boldsymbol{r}_{N} \sigma_{N}\right)$ and $\Omega$ is the simulation cell volume. The simulation cell for a quasi-2D system is subject to periodic boundary conditions in two dimensions; in the third, it has infinite extent.

Using the fact that

$$
\int_{\Omega} \mathrm{d} \boldsymbol{r}^{\prime} n_{\mathrm{XC}}\left(\boldsymbol{r}, \boldsymbol{r}^{\prime}\right)=-1,
$$

the definitions above reduce equation (1) to the form

$$
\begin{aligned}
E_{\mathrm{e}-\mathrm{e}}^{\mathrm{EW}}= & \frac{1}{2} \int_{\Omega} \mathrm{d} \boldsymbol{r} \int_{\Omega} \mathrm{d} \boldsymbol{r}^{\prime} n(\boldsymbol{r}) n\left(\boldsymbol{r}^{\prime}\right) v_{\mathrm{E}}\left(\boldsymbol{r}-\boldsymbol{r}^{\prime}\right) \\
& \quad+\frac{1}{2} \int_{\Omega} \mathrm{d} \boldsymbol{r} \int_{\Omega} \mathrm{d} \boldsymbol{r}^{\prime} n(\boldsymbol{r}) n_{\mathrm{XC}}\left(\boldsymbol{r}, \boldsymbol{r}^{\prime}\right)\left[v_{\mathrm{E}}\left(\boldsymbol{r}-\boldsymbol{r}^{\prime}\right)-\xi\right] \\
= & U_{\mathrm{Ha}}+U_{\mathrm{XC}}^{\mathrm{EW}} .
\end{aligned}
$$

Equation (6) illustrates the decomposition of the Coulomb energy into Hartree and exchange-correlation terms. In the MPC [1], this energy is replaced by

$$
\begin{aligned}
E_{\mathrm{e}-\mathrm{e}}^{\mathrm{MPC}}= & \left\langle\sum_{i>j} f\left(\boldsymbol{r}_{i j}\right)\right\rangle+\left\langle\sum_{i} \frac{1}{2} \int_{\Omega} \mathrm{d} \boldsymbol{r} n(\boldsymbol{r})\left[v_{\mathrm{E}}\left(\boldsymbol{r}-\boldsymbol{r}_{i}\right)-f\left(\boldsymbol{r}-\boldsymbol{r}_{i}\right)\right]\right\rangle \\
= & \frac{1}{2} \int_{\Omega} \mathrm{d} \boldsymbol{r} \int_{\Omega} \mathrm{d} \boldsymbol{r}^{\prime} n(\boldsymbol{r}) n\left(\boldsymbol{r}^{\prime}\right) v_{\mathrm{E}}\left(\boldsymbol{r}-\boldsymbol{r}^{\prime}\right) \\
& \quad+\frac{1}{2} \int_{\Omega} \mathrm{d} \boldsymbol{r} \int_{\Omega} \mathrm{d} \boldsymbol{r}^{\prime} n(\boldsymbol{r}) n_{\mathrm{XC}}\left(\boldsymbol{r}, \boldsymbol{r}^{\prime}\right) f\left(\boldsymbol{r}-\boldsymbol{r}^{\prime}\right) \\
= & U_{\mathrm{Ha}}+U_{\mathrm{XC}}^{\mathrm{MPC}} .
\end{aligned}
$$

Here $f(\boldsymbol{r})$ is the normal $1 / r$ Coulomb interaction, except that the vector $\boldsymbol{r}$ is reduced into the Wigner-Seitz cell of the simulation cell lattice. This is known as the minimum-image convention; it ensures that the interaction remains periodic. By comparing equations 
(6) and (7), it may be seen that the effect of using the MPC is to replace $\left(v_{\mathrm{E}}-\xi\right)$ with $f$ in the exchange-correlation term while leaving the Hartree term unchanged. This is desirable because the exchange-correlation hole should not be duplicated outside the simulation cell; the Ewald interaction $v_{\mathrm{E}}$ builds in the effects of this duplication, and therefore should not appear in $U_{\mathrm{XC}}$. This analysis applies equally to quasi-2D or fully 3D systems. Equation (7) gives the required energy expectation value. To obtain the corresponding term in the Hamiltonian, consider the expectation value of the total energy obtained using the Ewald interaction:

$$
E[\Psi]=\langle\Psi|\hat{H}| \Psi\rangle=E_{0}[\Psi]+E_{\mathrm{e}-\mathrm{e}}^{\mathrm{EW}}[\Psi]
$$

$E_{0}$, by definition, contains all terms except the electron-electron interaction. Replacing $E_{\mathrm{e}-\mathrm{e}}^{\mathrm{EW}}$ with $E_{\mathrm{e}-\mathrm{e}}^{\mathrm{MPC}}$ and solving $\delta(E[\Psi]-\lambda\langle\Psi \mid \Psi\rangle)=0$ gives

$$
\hat{H}_{\mathrm{e}-\mathrm{e}}^{\mathrm{MPC}}=\sum_{i>j} f\left(\boldsymbol{r}_{i j}\right)+\sum_{i} \int_{\Omega} \mathrm{d} \boldsymbol{r} n(\boldsymbol{r})\left[v_{\mathrm{E}}\left(\boldsymbol{r}-\boldsymbol{r}_{i}\right)-f\left(\boldsymbol{r}-\boldsymbol{r}_{i}\right)\right] .
$$

Note that the functional differentiation has removed the factor of $\frac{1}{2}$ in the second term. The relationship between $E_{\mathrm{e}-\mathrm{e}}^{\mathrm{MPC}}$ and $\hat{H}_{\mathrm{e}-\mathrm{e}}^{\mathrm{MPC}}$ is therefore

$$
E_{\mathrm{e}-\mathrm{e}}^{\mathrm{MPC}}=\left\langle\hat{H}_{\mathrm{e}-\mathrm{e}}^{\mathrm{MPC}}\right\rangle-\frac{1}{2} \int_{\Omega} \mathrm{d} \boldsymbol{r} \int_{\Omega} \mathrm{d} \boldsymbol{r}^{\prime} n(\boldsymbol{r}) n\left(\boldsymbol{r}^{\prime}\right)\left[v_{\mathrm{E}}\left(\boldsymbol{r}-\boldsymbol{r}^{\prime}\right)-f\left(\boldsymbol{r}-\boldsymbol{r}^{\prime}\right)\right] .
$$

To evaluate $E_{\mathrm{e}-\mathrm{e}}^{\mathrm{MPC}}$ or $\hat{H}_{\mathrm{e}-\mathrm{e}}^{\mathrm{MPC}}$ during a simulation requires a knowledge of $n(\boldsymbol{r})$, the electron density. In general, this is not known exactly before the simulation. However, a good approximation may be obtained from an independent-particle calculation; such calculations are routinely used to generate trial wave functions for use in QMC, so an approximate electron density is available. It may be shown [9] that the energy error in this approximation is $\mathrm{O}\left[(\Delta n)^{2}\right]$, where $\Delta n$ is the error in the density.

Equation (9) illustrates the reason for the improvement in speed achieved by the MPC. Two-body interactions require $\mathrm{O}\left[N^{2}\right]$ operations, while one-body interactions require only $\mathrm{O}[N]$; the only two-body interaction in the MPC is $f$, which is a much simpler function to evaluate than the costly $v_{\mathrm{E}}$. The remaining term in equation (9) is effectively a one-body potential. Using the 3D Fourier transforms

$$
\begin{aligned}
n_{\boldsymbol{k}} & =\frac{1}{\Omega} \int_{\Omega} \mathrm{d} \boldsymbol{r} n(\boldsymbol{r}) e^{\mathrm{i} \boldsymbol{k} \cdot \boldsymbol{r}} \\
g_{\boldsymbol{k}} & =\frac{1}{\Omega} \int_{\Omega} \mathrm{d} \boldsymbol{r}\left[v_{\mathrm{E}}(\boldsymbol{r})-f(\boldsymbol{r})\right] e^{\mathrm{i} \boldsymbol{k} \cdot \boldsymbol{r}},
\end{aligned}
$$

this term may be evaluated as

$$
\Omega \sum_{i} \sum_{\boldsymbol{k}} n_{\boldsymbol{k}}^{*} g_{\boldsymbol{k}} e^{\mathrm{i} \boldsymbol{k} \cdot \boldsymbol{r}_{i}}
$$

Since the transforms can be pre-calculated, the one-body term is also inexpensive. The use of 3D transforms means that the simulation cell may no longer have infinite extent in the non-periodic direction. To avoid overlapping, the electron density must also be restricted to a range $w$, where the size of the simulation cell in this direction is at least $2 w$. The requirement of finite extent is not unreasonable for quasi-2D systems, and the electron density usually tends exponentially to zero beyond a certain point. 


\section{Results}

All the results presented in this section are for a system of electrons moving in the potential of a positive background charge. This background charge has uniform density inside the slab and zero outside, thus defining the slab width. The simulation cell was chosen to be of square cross-section, with the size determined by the number of electrons used. The system is not spin-polarized.

The chosen slab width was 18.63 a.u., with background charge density $\left(4 / 3 \pi r_{s}^{3}\right)$ where $r_{s}=2.07$ a.u. This corresponds approximately to the electron density of aluminium.

The QMC simulations were carried out using either variational or fixed-node diffusion Monte Carlo [10], with trial wave functions obtained from density-functional theory calculations in the local density approximation. These trial wave functions were also used to calculate the exchange energy [11]

$$
E_{\mathrm{X}}=-\sum_{n} \sum_{n^{\prime}} \int_{\Omega} \mathrm{d} \boldsymbol{r} \int_{\Omega} \mathrm{d} \boldsymbol{r}^{\prime}\left[v_{\mathrm{E}}\left(\boldsymbol{r}-\boldsymbol{r}^{\prime}\right)-\xi\right] \phi_{n}^{*}(\boldsymbol{r}) \phi_{n}\left(\boldsymbol{r}^{\prime}\right) \phi_{n^{\prime}}(\boldsymbol{r}) \phi_{n^{\prime}}^{*}\left(\boldsymbol{r}^{\prime}\right)
$$

Here, $\phi_{n}$ represents the $n$th single-electron wave function. The many-electron trial function used in the QMC calculations was of the form

$$
\Psi(\boldsymbol{X})=e^{J(\boldsymbol{X})} D_{\uparrow}\left(\boldsymbol{r}_{1} \ldots \boldsymbol{r}_{N / 2}\right) D_{\downarrow}\left(\boldsymbol{r}_{N / 2+1} \ldots \boldsymbol{r}_{N}\right)
$$

where $D_{\uparrow}$ and $D_{\downarrow}$ are Slater determinants of the $\phi_{n}$. The up- and down-spin determimants are identical. The Jastrow factor has the following simple form:

$$
\begin{aligned}
& J(\boldsymbol{X})=-\sum_{i>j} u_{\sigma_{i} \sigma_{j}}\left(r_{i j}\right)+\sum_{i} \chi\left(r_{i}\right) \\
& u_{\sigma_{i} \sigma_{j}}\left(r_{i j}\right)=\frac{A}{r_{i j}}\left[1-\exp \left(-r_{i j} / F_{\sigma_{i} \sigma_{j}}\right)\right] \exp \left(-r_{i j}^{2} / L_{0}^{2}\right) \\
& \chi\left(r_{i}\right)=\sum_{k} c_{k} \sin \left(k z_{i}\right)
\end{aligned}
$$

The variational parameters here are $c_{k}$ and $A: F$ is related to $A$ by the cusp conditions $[12,13] ; L_{0}$ is chosen to ensure that $u$ decays to zero before $r_{i j}$ reaches the size of the simulation cell. Note that the centre of the slab corresponds to $z=w$, where the length of the simulation cell is $2 w ; \chi$ in equation (18) is symmetric about the slab centre.

Figures 2 and 3 show the dependence of the total energy per electron on system size for both the Ewald and MPC interactions. These results have been corrected for independent-particle finite-size errors [14]. The 3D MPC interaction has been shown to have significantly smaller finite-size errors than the 3D Ewald [1]. In contrast, Figures 2 and 3 illustrate that the Q2D version does not lead to a similar improvement. In fact, there is good agreement between the results obtained using the different interactions. The reasons for this are discussed in section 4. The slow convergence of the QMC results with system size is reflected in the exchange energy, calculated using equation (14) and also plotted in Figure 2.

The MPC does show a significant improvement in calculation time, which is illustrated in Figure 4. The figure clearly shows the $\mathrm{O}\left[N^{2}\right]$ scaling of the Ewald 


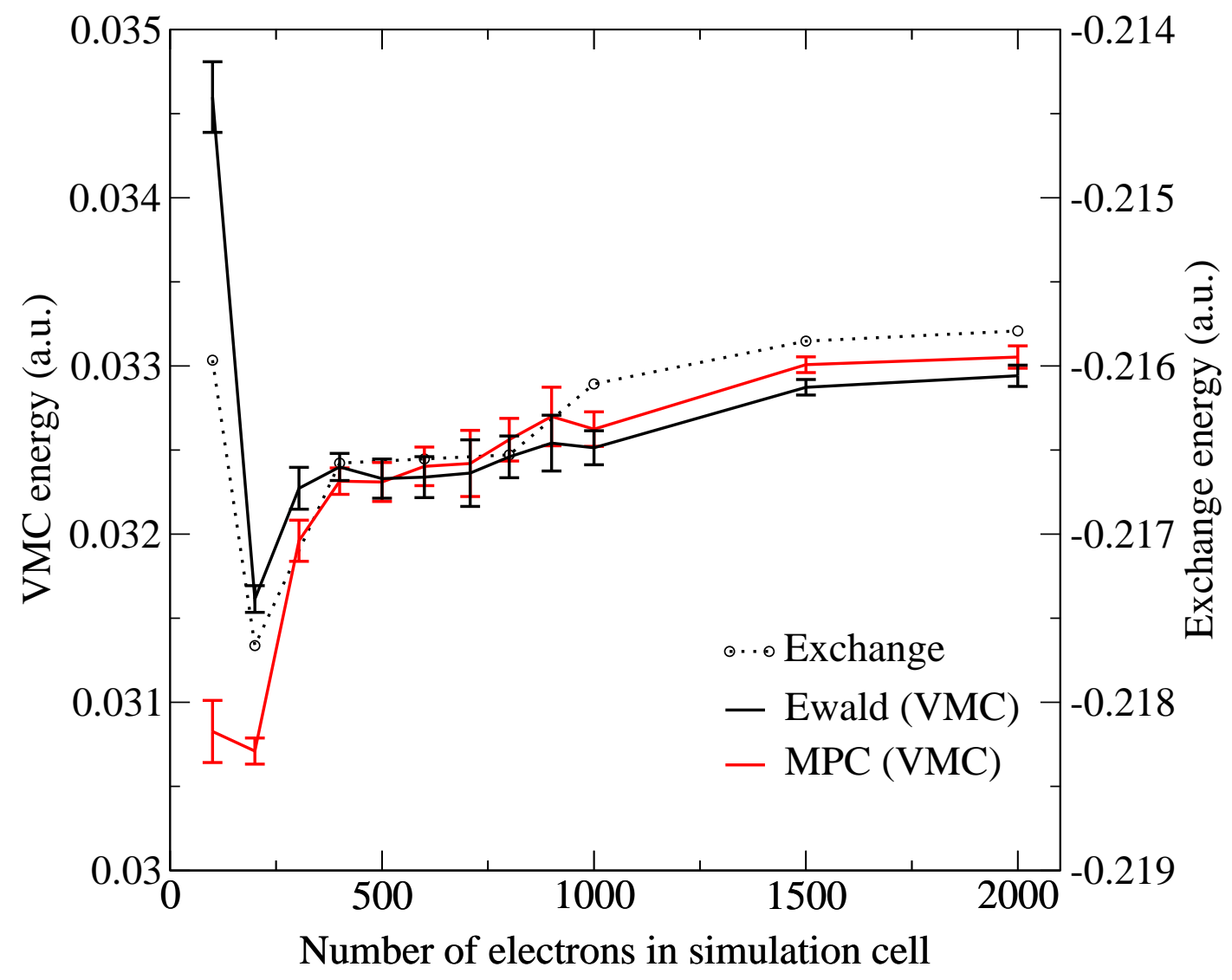

Figure 2. The total VMC energy per electron is plotted as a function of system size, comparing the Ewald and MPC interactions. The results have been corrected to account for independent-particle finite-size errors [1]. The exchange energy is also plotted, with a shift in the energy scale. Error bars are not shown for the exchange energy as the results are fully converged with respect to the number of $\boldsymbol{k}$-vectors used to represent the single-electron wave function. Any remaining error is a consequence of the quality of the LDA-DFT wave functions (and is therefore difficult to estimate).

interaction. The MPC interaction should also be dominated by the $\mathrm{O}\left[N^{2}\right]$ term for large system sizes, but the function being evaluated is much less costly. For the system sizes tested here, the $\mathrm{O}[N]$ term also contributes significantly. The time required for the MPC pre-calculation is not included in the figure; for any serious simulation, it is negligible in comparison with the time spent calculating the electron-electron interaction during the simulation. In our DMC simulations, evaluating the electron-electron interaction with the Ewald method takes up a significant fraction of the calculation time (often 40-50\%). Figure 4 shows that the MPC interaction is nearly two orders of magnitude faster than the Ewald. This is a much more dramatic improvement than in the $3 \mathrm{D}$ case.

\section{Conclusions}

The results of section 3 demonstrate that although the MPC interaction enjoys the expected advantage in speed over the Ewald sum, it does not reduce the finite-size 


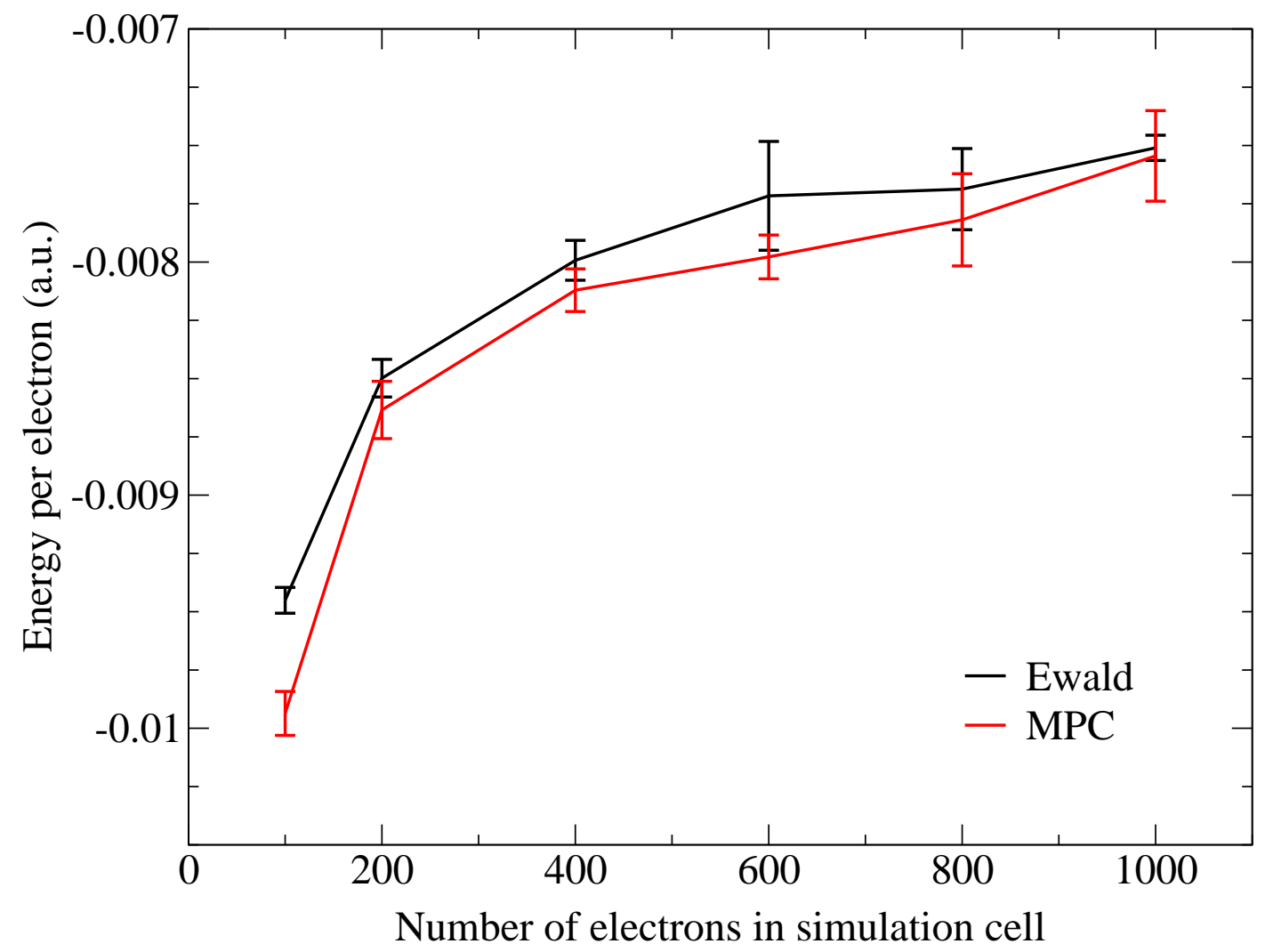

Figure 3. The total energy per electron as a function of system size, obtained in fixed-node DMC. Independent-particle finite-size error corrections have been applied.

errors. In order to understand this, it is instructive to consider the expected size of the finite-size error incurred by using the Ewald interaction:

$E_{\mathrm{XC}}^{\mathrm{EW}}-E_{\mathrm{XC}}^{\mathrm{MPC}}=\frac{1}{2} \int_{\Omega} \mathrm{d} \boldsymbol{r} \int_{\Omega} \mathrm{d} \boldsymbol{r}^{\prime} n(\boldsymbol{r}) n_{\mathrm{XC}}\left(\boldsymbol{r}, \boldsymbol{r}^{\prime}\right)\left[v_{\mathrm{E}}\left(\boldsymbol{r}-\boldsymbol{r}^{\prime}\right)-\xi-f\left(\boldsymbol{r}-\boldsymbol{r}^{\prime}\right)\right]$.

The exchange-correlation hole described by $n_{\mathrm{XC}}$ is generally short-ranged. An approximation to equation (19) may therefore be obtained by using the small- $r$ and large- $L$ expansion

$$
v_{\mathrm{E}}(\boldsymbol{r})-\xi=\frac{1}{r}-\frac{C}{L^{3}}\left(z^{2}-\frac{r_{\|}^{2}}{2}\right)+\mathrm{O}\left[r^{4}\right]+\mathrm{O}\left[e^{-L^{2} / \sigma^{2}}\right] .
$$

which is derived in Appendix B. Here $L$ is the simulation cell lattice parameter, $C$ is a constant and $\sigma$ is the Ewald convergence parameter described in Appendix A.

Combining equations (19) and (20) gives an approximation for the expected error incurred by using the quasi-2D Ewald sum:

$$
-\frac{C}{2 L^{3}} \int_{\Omega} \mathrm{d} \boldsymbol{r} \int_{\Omega} \mathrm{d} \boldsymbol{r}^{\prime} n(\boldsymbol{r}) n_{\mathrm{XC}}\left(\boldsymbol{r}, \boldsymbol{r}^{\prime}\right)\left[\left(z-z^{\prime}\right)^{2}-\frac{1}{2}\left(\boldsymbol{r}_{\|}-\boldsymbol{r}_{\|}^{\prime}\right)^{2}\right] .
$$

Thus, a large cancellation of the error may be anticipated in regions where the parallel and perpendicular directions are equivalent; this is the case in the bulk of the system, where the exchange hole is roughly spherical. At the surfaces of the system this is no 


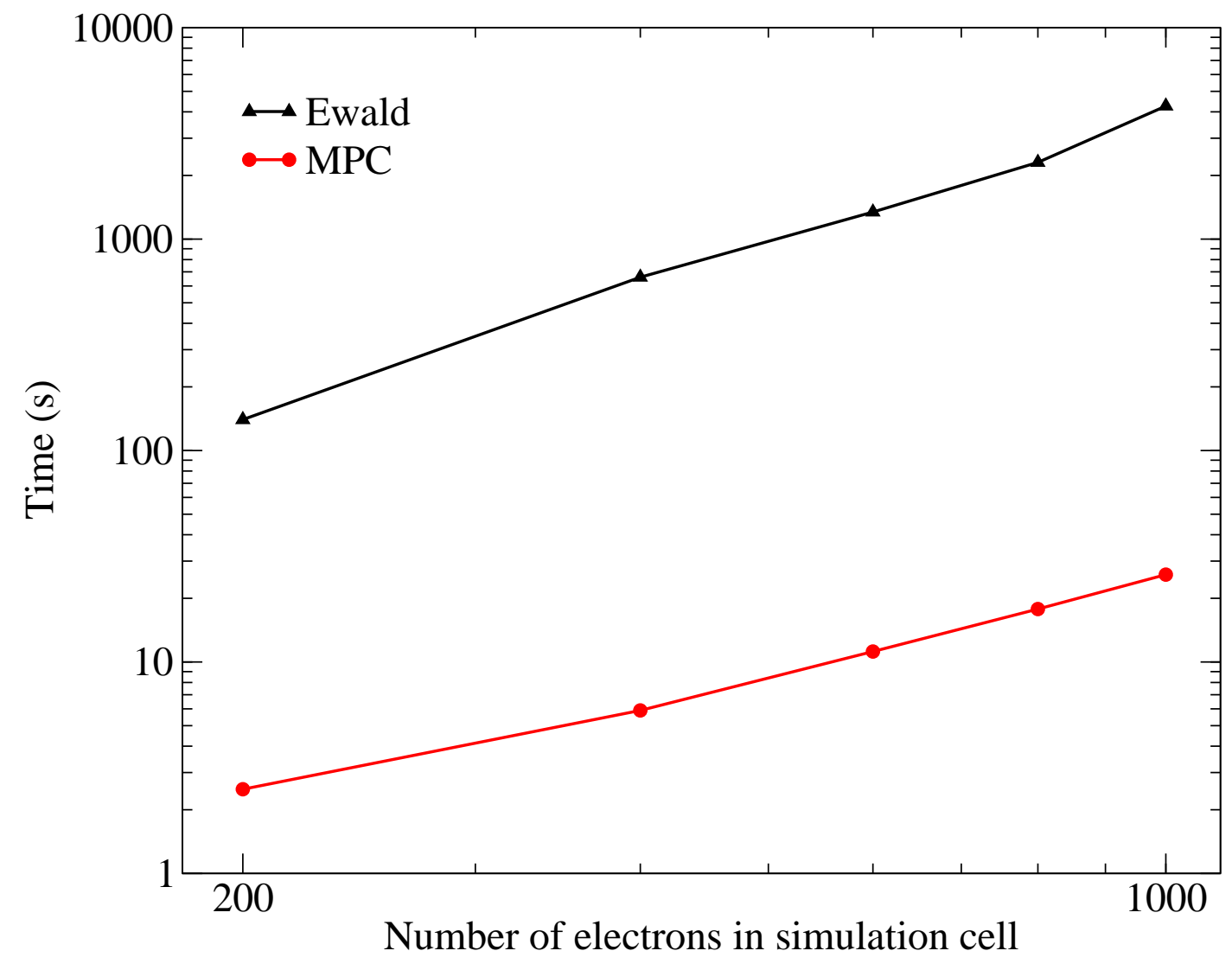

Figure 4. The time taken to calculate the electron-electron interaction during an 8000-move variational quantum Monte Carlo simulation, comparing the Ewald and MPC interactions.

longer true. However, it is also likely that the exchange hole expands in these regions, making the small-r expansion used in equation (21) less appropriate.

In 3D, the small- $r$ expansion of the Ewald interaction is spherically-symmetric [3] and there is no error cancellation.

The slow convergence of the QMC and exchange energies suggests that a successful supercell calculation of the surface energy would require the use of large systems. This may be one reason for the current discrepancy between QMC estimates of this quantity and those obtained by other (non-supercell) methods. However, further investigation is required.

\section{Appendix A. Derivation of the Ewald Sum for Quasi-2D Systems}

The generalization of the Ewald summation to a system with periodic repeat in only two dimensions was first obtained by Parry [4, 5]. However, this original derivation, although it leads to the correct result, is difficult to follow. An alternative derivation is presented here.

The problem is to find the potential due to a charge of unit magnitude at the origin 
and all its images in the plane. The charge distribution is therefore

$$
\rho(\boldsymbol{r})=\sum_{\boldsymbol{R}} \delta(\boldsymbol{r}-\boldsymbol{R})
$$

where $\boldsymbol{R}$ is a $2 \mathrm{D}$ lattice vector. The Ewald method [2] is to rewrite the charge distribution, creating a smooth term which may be evaluated in reciprocal space and a rapidly-decaying term which converges quickly in real space. In the quasi-2D system, this is modified slightly, and the charge distribution is in fact rewritten as

$$
\begin{aligned}
\rho(\boldsymbol{r})= & \left(\sum_{\boldsymbol{R}}\left[\delta(\boldsymbol{r}-\boldsymbol{R})-\frac{1}{\pi \sqrt{\pi} \sigma^{3}} e^{-(\boldsymbol{r}-\boldsymbol{R})^{2} / \sigma^{2}}\right]\right) \\
& +\left(\sum_{\boldsymbol{R}} \frac{1}{\pi \sqrt{\pi} \sigma^{3}} e^{-(\boldsymbol{r}-\boldsymbol{R})^{2} / \sigma^{2}}-\frac{1}{\sqrt{\pi} \sigma A} e^{-z^{2} / \sigma^{2}}\right) \\
& +\left(\frac{1}{\sqrt{\pi} \sigma A} e^{-z^{2} / \sigma^{2}}\right) \\
= & \rho_{1}(\boldsymbol{r})+\rho_{2}(\boldsymbol{r})+\rho_{3}(\boldsymbol{r}) .
\end{aligned}
$$

Here, $A$ is the area of the $2 \mathrm{D}$ cell defined by the primitive lattice vectors and $\sigma$ is a parameter which may be adjusted to assure the rapid convergence of real and reciprocal space sums (without affecting the result). The three terms on the right-hand side of equation (A.2) will be dealt with separately, starting with $\rho_{1}$.

The contribution to the potential from each term in the sum decays rapidly with $|\boldsymbol{r}-\boldsymbol{R}|$; the potential is therefore evaluated in real space, using Gauss' Law. Consider the charge distribution

$$
\rho(\boldsymbol{r})=\delta(\boldsymbol{r})-\frac{1}{\pi \sqrt{\pi} \sigma^{3}} e^{-\boldsymbol{r}^{2} / \sigma^{2}} .
$$

By Gauss' Law, the electric field generated by this potential has magnitude

$$
E(r)=\frac{2 e^{-r^{2} / \sigma^{2}}}{\sqrt{\pi} \sigma r}+\frac{1}{r^{2}} \operatorname{erfc}\left(\frac{r}{\sigma}\right)
$$

in atomic units. Insisting that the potential must tend to zero as $r \rightarrow \infty$ gives

$$
\begin{aligned}
\phi(r) & =\int_{r}^{\infty} \mathrm{d} r^{\prime} E\left(r^{\prime}\right) \\
& =\frac{1}{r} \operatorname{erfc}\left(\frac{r}{\sigma}\right)
\end{aligned}
$$

so that the contribution to the potential from the charge distribution $\rho_{1}$ is

$$
\phi_{1}(\boldsymbol{r})=\sum_{\boldsymbol{R}} \frac{1}{|\boldsymbol{r}-\boldsymbol{R}|} \operatorname{erfc}\left(\frac{|\boldsymbol{r}-\boldsymbol{R}|}{\sigma}\right) .
$$

The second contribution comes from a reciprocal space sum; the charge distribution is therefore rewritten as

$$
\rho_{2}(\boldsymbol{r})=e^{-z^{2} / \sigma^{2}} \sum_{\boldsymbol{k}} \rho_{\boldsymbol{k}} e^{\mathrm{i} \boldsymbol{k} \cdot \boldsymbol{r}_{\|}}
$$


where $\boldsymbol{k}$ is the set of $2 \mathrm{D}$ reciprocal lattice vectors and the $\rho_{\boldsymbol{k}}$ are Fourier coefficients. The sum excludes $\boldsymbol{k}=\mathbf{o}$, since $\rho_{\boldsymbol{k}=\mathbf{o}}=0$ by design. The other coefficients are given by

$$
\begin{aligned}
\rho_{\boldsymbol{k}} & =\frac{1}{A} \int_{\text {cell }} \mathrm{d} \boldsymbol{r}_{\|} \sum_{\boldsymbol{R}} \frac{1}{\sqrt{\pi} \pi \sigma^{3}} e^{-\left(\boldsymbol{r}_{\|}-\boldsymbol{R}\right)^{2} / \sigma^{2}} e^{-\mathrm{i} \boldsymbol{k} \cdot \boldsymbol{r}_{\|}} \\
& =\frac{1}{A \sqrt{\pi} \pi \sigma^{3}} \int_{\text {space }} \mathrm{d} \boldsymbol{r}_{\|} e^{-\boldsymbol{r}_{\|}^{2} / \sigma^{2}-\mathrm{i} \boldsymbol{k} \cdot \boldsymbol{r}_{\|}} \\
& =\frac{1}{A \sqrt{\pi} \sigma} e^{-k^{2} \sigma^{2} / 4} .
\end{aligned}
$$

The desired potential is expressed as a similar series:

$$
\phi_{2}(\boldsymbol{r})=\sum_{\boldsymbol{k}} \phi_{\boldsymbol{k}}(z) e^{i \boldsymbol{k} \cdot \boldsymbol{r}_{\|}}
$$

These expressions may then be subsituted into Poisson's equation,

$$
\nabla^{2} \phi_{2}(\boldsymbol{r})=-4 \pi \rho_{2}(\boldsymbol{r})
$$

to give an equation for the coefficients $\phi_{\boldsymbol{k}}(z)$ :

$$
\left(\frac{d^{2}}{d z^{2}}-k^{2}\right) \phi_{\boldsymbol{k}}(z)=-\frac{4 \sqrt{\pi}}{\sigma A} e^{-\left(z^{2}+k^{2} / 4\right) / \sigma^{2}} .
$$

This may be solved with the Green's function

$$
G_{\boldsymbol{k}}\left(z, z^{\prime}\right)=-\frac{1}{2 k} e^{-k\left|z-z^{\prime}\right|}
$$

to give

$$
\phi_{\boldsymbol{k}}(z)=-\int_{-\infty}^{\infty} \mathrm{d} z^{\prime} \frac{1}{2 k} e^{-k\left|z-z^{\prime}\right|}\left(-\frac{4 \sqrt{\pi}}{\sigma A} e^{-\left(z^{2}+k^{2} / 4\right) / \sigma^{2}}\right),
$$

which, after integration, yields the potential

$\phi_{2}(\boldsymbol{r})=\frac{\pi}{A} \sum_{\boldsymbol{k}} \frac{1}{k}\left[e^{-k z} \operatorname{erfc}\left(\frac{\sigma k}{2}-\frac{z}{\sigma}\right) e^{k z} \operatorname{erfc}\left(\frac{\sigma k}{2}+\frac{z}{\sigma}\right)\right] e^{\mathrm{i} \boldsymbol{k} \cdot \boldsymbol{r}_{\|}}$.

Finally, the third charge distribution is

$$
\rho_{3}(\boldsymbol{r})=\frac{1}{A \sqrt{\pi} \sigma} e^{-z^{2} / \sigma^{2}} .
$$

Since this function only depends on $z$, Poisson's equation reduces to a one-dimensional problem; the appropriate Green's function is

$$
G\left(z, z^{\prime}\right)=\frac{1}{2}\left|z-z^{\prime}\right| .
$$

The potential is therefore given by

$$
\begin{aligned}
\phi_{3}(\boldsymbol{r}) & =-4 \pi \int_{-\infty}^{\infty} \mathrm{d} z^{\prime}\left[\frac{1}{2}\left|z-z^{\prime}\right|\right)\left(\frac{1}{A \sqrt{\pi} \sigma} e^{-z^{\prime 2} / \sigma^{2}}\right] \\
& =-\frac{2 \pi}{A}\left(z \operatorname{erf}\left(\frac{z}{\sigma}\right)+\frac{\sigma}{\sqrt{\pi}} e^{-z^{2} / \sigma^{2}}\right) .
\end{aligned}
$$


Combining the three previous results gives the following expression for the potential due to a charge at $\boldsymbol{r}=\mathbf{o}$ and the corresponding images:

$$
\begin{aligned}
v_{\mathrm{E}}(\boldsymbol{r})= & \sum_{\boldsymbol{R}} \frac{1}{|\boldsymbol{r}-\boldsymbol{R}|} \operatorname{erfc}\left(\frac{|\boldsymbol{r}-\boldsymbol{R}|}{\sigma}\right)-\frac{2 \pi}{A}\left[z \operatorname{erf}\left(\frac{z}{\sigma}\right)+\frac{\sigma}{\sqrt{\pi}} e^{-z^{2} / \sigma^{2}}\right] \\
& +\sum_{\boldsymbol{k}} \frac{\pi}{k A}\left[e^{-k z} \operatorname{erfc}\left(\frac{\sigma k}{2}-\frac{z}{\sigma}\right)+e^{k z} \operatorname{erfc}\left(\frac{\sigma k}{2}+\frac{z}{\sigma}\right)\right] e^{\mathrm{i} \boldsymbol{k} \cdot \boldsymbol{r}_{\|}} .
\end{aligned}
$$

The self-interaction energy is the energy associated with the interaction between this charge and its images:

$$
\begin{aligned}
\xi & =\lim _{\boldsymbol{r} \rightarrow \mathbf{o}}\left(v_{\mathrm{E}}(\boldsymbol{r})-\frac{1}{r}\right) \\
& =\lim _{\boldsymbol{r} \rightarrow \mathbf{o}}\left(\frac{1}{r} \operatorname{erfc}\left(\frac{r}{\sigma}\right)-\frac{1}{r}\right)+\sum_{\boldsymbol{R} \neq \mathbf{o}} \frac{1}{R} \operatorname{erfc}\left(\frac{R}{\sigma}\right)-\frac{2 \sigma \sqrt{\pi}}{A}+\sum_{\boldsymbol{k} \neq \mathbf{o}} \frac{2 \pi}{k A} \operatorname{erfc}\left(\frac{\sigma k}{2}\right) \\
& =-\frac{2}{\sigma \sqrt{\pi}}+\sum_{\boldsymbol{R} \neq \mathbf{o}} \frac{1}{R} \operatorname{erfc}\left(\frac{R}{\sigma}\right)-\frac{2 \sigma \sqrt{\pi}}{A}+\sum_{\boldsymbol{k} \neq \mathbf{o}} \frac{2 \pi}{k A} \operatorname{erfc}\left(\frac{\sigma k}{2}\right) .
\end{aligned}
$$

\section{Appendix B. Expansion of the Quasi-2D Ewald Interaction}

The function appearing in the exchange-correlation energy $\left(U_{\mathrm{XC}}^{\mathrm{EW}}\right.$ in equation $\left.(6)\right)$ is $v_{\mathrm{E}}(\boldsymbol{r})-\xi$. The exchange-correlation hole is normally short-ranged; the extent to which $v_{\mathrm{E}}(\boldsymbol{r})-\xi$ deviates from $1 / r$ at small $r$ may be used to estimate the finite-size error associated with the Ewald interaction. The expansion of this function for small $r$ and large lattice parameter $(L)$ follows.

Combining equations (A.19) and (A.18) gives:

$$
\begin{aligned}
v_{\mathrm{E}}(\boldsymbol{r})-\xi= & \frac{2}{\sigma \sqrt{\pi}}-\sum_{\boldsymbol{R} \neq \mathbf{o}} \frac{\operatorname{erfc}\left(\frac{R}{\sigma}\right)}{R}+\sum_{\boldsymbol{R}} \frac{\operatorname{erfc}\left(\frac{|\boldsymbol{r}-\boldsymbol{R}|}{\sigma}\right)}{|\boldsymbol{r}-\boldsymbol{R}|} \\
& -\frac{2 \pi}{A}\left[z \operatorname{erf}\left(\frac{z}{\sigma}\right)+\frac{\sigma}{\sqrt{\pi}}\left(e^{-z^{2} / \sigma^{2}}-1\right)\right] \\
& +\sum_{\boldsymbol{k} \neq \mathbf{o}} \frac{\pi}{k A}\left\{\left[e^{-k z} \operatorname{erfc}\left(\frac{\sigma k}{2}-\frac{z}{\sigma}\right)+e^{k z} \operatorname{erfc}\left(\frac{\sigma k}{2}+\frac{z}{\sigma}\right)\right] \cos \boldsymbol{k} \cdot \boldsymbol{r}_{\|}\right. \\
& \left.-2 \operatorname{erfc}\left(\frac{\sigma k}{2}\right)\right\} .
\end{aligned}
$$

The first line of this expression reduces quickly to

$$
\frac{1}{r}+\frac{2 r^{2}}{3 \sigma^{3} \sqrt{\pi}}+\mathrm{O}\left[r^{4}\right]+\mathrm{O}\left[e^{-L^{2} / \sigma^{2}}\right]
$$

The second line is also simply expanded, giving

$$
-\frac{2 \sqrt{\pi} z^{2}}{\sigma A}+\mathrm{O}\left[z^{4}\right]
$$

The sum in $\boldsymbol{k}$-space is slightly more involved. To begin, we note that

$$
\operatorname{erfc}\left(x_{0}+x\right)=\operatorname{erfc}\left(x_{0}\right)+\frac{2}{\sqrt{\pi}}\left(x^{2} x_{0}-x\right) e^{-x_{0}^{2}}+\mathrm{O}\left[x^{3}\right] \text {. }
$$


Applying this result,

$$
\begin{aligned}
e^{-k z} \operatorname{erfc}\left(\frac{\sigma k}{2}-\frac{z}{\sigma}\right)+e^{k z} \operatorname{erfc}\left(\frac{\sigma k}{2}+\frac{z}{\sigma}\right)= & \left(2+k^{2} z^{2}\right) \operatorname{erfc}\left(\frac{\sigma k}{2}\right) \\
& -\frac{2 z^{2} k}{\sigma \sqrt{\pi}} e^{-(\sigma k / 2)^{2}}+\mathrm{O}\left[z^{4}\right] .
\end{aligned}
$$

The error is of order $z^{4}$ rather than $z^{3}$ because any terms involving odd powers of $z$ must cancel out. The next step is to expand the cosine to $\mathrm{O}\left[r^{2}\right]$; the $\boldsymbol{k}$-space sum of equation (B.1) then becomes

$\sum_{\boldsymbol{k} \neq \mathbf{o}}\left[\frac{\pi k}{A}\left(z^{2}-\frac{\left(\boldsymbol{k} \cdot \boldsymbol{r}_{\|}\right)^{2}}{k^{2}}\right) \operatorname{erfc}\left(\frac{\sigma k}{2}\right)-\frac{2 \sqrt{\pi} z^{2}}{\sigma A} e^{-(\sigma k / 2)^{2}}\right]+\mathrm{O}\left[r^{4}\right]$

which reduces to

$$
\sum_{\boldsymbol{k} \neq \mathbf{o}}\left[\frac{\pi k}{A}\left(z^{2}-\frac{k^{2} r_{\|}^{2}}{2 k^{2}}\right) \operatorname{erfc}\left(\frac{\sigma k}{2}\right)-\frac{2 \sqrt{\pi} z^{2}}{\sigma A} e^{-(\sigma k / 2)^{2}}\right]+\mathrm{O}\left[r^{4}\right]
$$

from a comparison of the contributions to the sum of all the $\boldsymbol{k}$-vectors of a given magnitude.

To proceed further, we use the following two-dimensional Fourier series:

$$
\sum_{\boldsymbol{R}} e^{-((\boldsymbol{r}-\boldsymbol{R}) / \sigma)^{2}}=\sum_{\boldsymbol{k}} \frac{\pi \sigma^{2}}{A} e^{-(\sigma k / 2)^{2}} e^{\mathrm{i} \boldsymbol{k} \cdot \boldsymbol{r}}
$$

with $\boldsymbol{r}=\mathbf{o}$. This gives

$$
\begin{aligned}
\sum_{\boldsymbol{k} \neq \mathbf{o}} e^{-(\sigma k / 2)^{2}} & =\frac{A}{\pi \sigma^{2}} \sum_{\boldsymbol{R}} e^{-(R / \sigma)^{2}}-1 \\
& =\frac{A}{\pi \sigma^{2}}-1+\mathrm{O}\left[e^{-L^{2} / \sigma^{2}}\right]
\end{aligned}
$$

which, when substituted back into equation (B.1), leads to

$$
v_{\mathrm{E}}(\boldsymbol{r})-\xi=\frac{1}{r}+\left(z^{2}-\frac{r_{\|}^{2}}{2}\right)\left(\sum_{\boldsymbol{k} \neq \mathbf{o}} \frac{\pi k}{A} \operatorname{erfc}\left(\frac{\sigma k}{2}\right)-\frac{4}{3 \sigma^{3} \sqrt{\pi}}\right)+\mathrm{O}\left[r^{4}\right]+\mathrm{O}\left[e^{-L^{2} / \sigma^{2}}\right] \text {. }
$$

The remaining $\boldsymbol{k}$-space sum may written in terms of the new variable $\beta=1 / \sigma^{2}$ :

$$
\begin{aligned}
\sum_{\boldsymbol{k} \neq \mathbf{o}} \frac{\pi k}{A} \operatorname{erfc}\left(\frac{\sigma k}{2}\right) & =\sum_{\boldsymbol{k}} \frac{\pi k}{A} \operatorname{erfc}\left(\frac{k}{2 \sqrt{\beta}}\right) \\
& =S(\beta) .
\end{aligned}
$$

Differentiating,

$$
\begin{aligned}
\frac{\mathrm{d} S}{\mathrm{~d} \beta} & =\sum_{\boldsymbol{k}} \frac{\sqrt{\pi}}{2 A \beta \sqrt{\beta}} k^{2} e^{-k^{2} / 4 \beta} \\
& =\frac{2 \sqrt{\pi} \sqrt{\beta}}{A} \frac{\mathrm{d}}{\mathrm{d} \beta}\left(\sum_{\boldsymbol{k}} e^{-k^{2} / 4 \beta}\right) \\
& =\frac{2 \sqrt{\pi} \sqrt{\beta}}{A} \frac{\mathrm{d}}{\mathrm{d} \beta}\left(\frac{A \beta}{\pi} \sum_{\boldsymbol{R}} e^{-R^{2} \beta}\right) \\
& =\frac{2 \sqrt{\beta}}{\sqrt{\pi}} \sum_{\boldsymbol{R}}\left(1-R^{2} \beta\right) e^{-R^{2} \beta} .
\end{aligned}
$$


Here, equation (B.8) has again been used to convert the reciprocal space sum to one in real space. To recover the original sum, we integrate from 0 to $\beta$ :

$$
\begin{aligned}
S(\beta)-S(0) & =\int_{0}^{\beta} \mathrm{d} \beta \frac{2 \sqrt{\beta}}{\sqrt{\pi}} \sum_{\boldsymbol{R}}\left(1-R^{2} \beta\right) e^{-R^{2} \beta} \\
& =\frac{2}{\sqrt{\pi}} \sum_{\boldsymbol{R}} \int_{0}^{\sqrt{\beta}} \mathrm{d} t 2 t\left(1-R^{2} t^{2}\right) e^{-R^{2} t^{2}} \\
& =\frac{4}{\sqrt{\pi}} \sum_{\boldsymbol{R} \neq \mathbf{o}}\left(I_{2}-R^{4} I_{4}\right)+\frac{4}{\sqrt{\pi}} \int_{0}^{\sqrt{\beta}} \mathrm{d} t t^{2} .
\end{aligned}
$$

The two remaining integrals are

$$
\begin{aligned}
I_{4} & =\int_{0}^{\sqrt{\beta}} \mathrm{d} t t^{4} e^{-R^{2} t^{2}} \\
& =-\frac{\beta \sqrt{\beta}}{2 R^{2}} e^{-R^{2} \beta}+\frac{3}{2 R^{2}} I_{2}
\end{aligned}
$$

and

$$
\begin{aligned}
I_{2} & =\int_{0}^{\sqrt{\beta}} \mathrm{d} t t^{2} e^{-R^{2} t^{2}} \\
& =-\frac{\sqrt{\beta}}{2 R^{2}} e^{-R^{2} \beta}+\frac{\sqrt{\pi}}{4 R^{3}} \operatorname{erf}(R \sqrt{\beta}) .
\end{aligned}
$$

Substituting these results into equation (B.13), and using the fact that $S(0)=0$,

$$
\begin{aligned}
S(\beta) & =\frac{4}{\sqrt{\pi}} \sum_{\boldsymbol{R} \neq \mathbf{o}}\left[\left(\frac{\sqrt{\beta}}{4 R^{2}}-\frac{\beta \sqrt{\beta}}{2}\right) e^{-R^{2} \beta}-\frac{\sqrt{\pi}}{8 R^{3}} \operatorname{erf}(R \sqrt{\beta})\right]+\frac{4 \beta \sqrt{\beta}}{3 \sqrt{\pi}} \\
& =\frac{4 \beta \sqrt{\beta}}{3 \sqrt{\pi}}-\frac{1}{2} \sum_{\boldsymbol{R} \neq \mathbf{o}} \frac{1}{R^{3}} \operatorname{erf}(R \sqrt{\beta})+\mathrm{O}\left[e^{-L^{2} \beta}\right] .
\end{aligned}
$$

Returning to the original summation,

$$
\begin{aligned}
\sum_{\boldsymbol{k} \neq \mathbf{o}} \frac{\pi k}{A} \operatorname{erfc}\left(\frac{\sigma k}{2}\right) & =\frac{4}{3 \sigma^{3} \sqrt{\pi}}-\frac{1}{2} \sum_{\boldsymbol{R} \neq \mathbf{o}} \frac{1}{R^{3}} \operatorname{erf}\left(\frac{R}{\sigma}\right)+\mathrm{O}\left[e^{-L^{2} / \sigma^{2}}\right] \\
& =\frac{4}{3 \sigma^{3} \sqrt{\pi}}-\frac{C}{L^{3}}+\mathrm{O}\left[e^{-L^{2} / \sigma^{2}}\right]
\end{aligned}
$$

where the constant $C$ is given by

$$
C=\frac{1}{2} \sum_{m, n=-\infty}^{\infty}\left(m^{2}+n^{2}\right)^{-3 / 2}
$$

and the prime here indicates that the term with $m=n=0$ should be excluded from the sum. It may be shown [15] that this sum reduces to

$$
4 \beta(3 / 2) \zeta(3 / 2)
$$

where $\beta$ and $\zeta$ are the Dirichlet beta and Riemann zeta functions respectively, giving $C=4.516810842$.

Combining equations (B.17) and (B.10) gives the final result:

$$
v_{\mathrm{E}}(\mathbf{r})-\xi=\frac{1}{r}-\frac{C}{L^{3}}\left(z^{2}-\frac{r_{\|}^{2}}{2}\right)+\mathrm{O}\left[r^{4}\right]+\mathrm{O}\left[e^{-L^{2} / \sigma^{2}}\right] .
$$




\section{References}

[1] Williamson A J, Rajagopal G, Needs R J, Fraser L M, Foulkes W M C, Wang Y and Chou M-Y 1997 Phys. Rev. B 55 R4851

[2] Tosi M P 1965 Solid State Physics: Advances in Research and Applications vol 16 (London: Academic)

[3] Fraser L M, Foulkes W M C, Rajagopal G, Needs R J, Kenny S D and Williamson A J 1996 Phys. Rev. B 531814

[4] Parry D E 1975 Surface Science 49433

[5] Parry D 1976 Surface Science $\mathbf{5 4} 195$

[6] Yan Z, Perdew J P, Kurth S, Fiolhais C and Almeida L 2000 Phys. Rev. B 612595

[7] Acioli P H and Ceperley D M 1996 Phys. Rev. B 5417199

[8] Li X-P, Needs R J, Martin R M and Ceperley D M 1992 Phys. Rev. B 456124

[9] Needs R J, Towler M D, Drummond, N D and Kent P R C 2003 CASINO Version 1.6.2 User Manual (University of Cambridge)

[10] Foulkes W M C, Mitas L, Needs R J and Rajagopal G 2001 Rev. Mod. Phys. 7333

[11] Szabo A and Ostlund N 1989 Modern Quantum Chemistry (New York: McGraw-Hill)

[12] Fahy S, Wang X W and Louie S G 1990 Phys. Rev. B 423503

[13] Kato T 1957 Commun. Pure Appl. Math. 10151

[14] Kent P R C, Hood R Q, Williamson A J, Needs R J, Foulkes, W M C and Rajagopal G 1999 Phys. Rev. B 591917

[15] Borwein J M and Borwein P B 1987 Pi and the AGM: A Study in Analytic Number Theory and Computational Complexity (New York: Wiley) 